\title{
Stress and wellbeing in urban college students in the U.S. during the COVID-19 pandemic: Can grit and gratitude help?
}

\author{
Giacomo Bono $\cdot$ Kresimir Reil $\cdot$ Jadwiga Hescox
}

\begin{abstract}
College is filled with opportunity, challenge and growth - as students expand their relationships and social capital, make formative life decisions, and overcome stress to achieve life goals. The current short-term longitudinal study started before campus closure due to the COVID19 pandemic and ended at the completion of the semester at an urban university. It investigates the stress and subjective wellbeing of freshmen, impacts of the pandemic on their psychological, academic and financial wellbeing and their resilience to the pandemic during this period, and the role of socioeconomic status. It also examines whether grit and gratitude helped in these areas. First, we compared students ( $\mathrm{N}=86$ freshmen) in terms of their parents' education level; high vs. low groups were created (i.e., completed high school or less vs. started or completed college up to graduate school). The low group reported significantly more perceived stress and subjective wellbeing than the high group prior to the pandemic. Next, examination of the two groups in terms of pandemic impacts and pandemic resilience revealed that the low group reported significantly more financial and academic impacts than the high group, but not more resilience or life event stress. Finally, we examined grit and gratitude prior to the pandemic and found that grit predicted significantly greater pandemic resilience and marginally lower psychological impact and that gratitude predicted significantly less impact to academic functioning at the end of the semester. However, the low parent education group also increased in grateful emotion, whereas the high parent group decreased, suggesting that gratitude helped the low group more during the pandemic. Overall results suggest that grit and gratitude can be promoted to protect college students' subjective wellbeing and better cope with adversity of the pandemic. The study closes with suggestions for intervention.
\end{abstract}

Keywords: Subjective wellbeing, stress, grit, gratitude, resilience, COVID-19, pandemic 


\section{Practice implications}

The current study occurred prior to and during the onset of a global pandemic that has disproportionately influenced minorities in the U.S. It compares how urban college students of lower versus higher socioeconomic status (SES) faired in terms of the pandemic's impacts on their lives and whether the two personality factors of grit and gratitude helped predict improved impact outcomes. It also examined whether the subjective wellbeing (SWB) of these two groups differed before and during the pandemic and whether it was improved by grit and gratitude. Results indicated that students of parents with education limited to high school face more stress, and less SWB than peers whose parents were college-educated, and they provide clues to better support students' mental health and wellbeing during adversities like the COVID-19 pandemic.

First, low parental education students did not differ from their high parent education counterparts in grit prior to the pandemic and in resilient responding to the pandemic. These findings suggest that these two behavioral strengths are common in equal supply to all incoming urban college students, regardless of SES, making intervention universally amenable. Research can explore how the sources and forms of grit and resilience vary among college students, though, to create more targeted intervention.

Second, that low parent education students were lower in gratitude prior to the pandemic but increased more during the pandemic, compared to high parent education students, has implications for improving gratitude intervention in two ways. Research should not only consider how to address the obstacles of greater daily stress and lower SWB among low SES students in creating more effective gratitude interventions in general, but it should also consider how to harness the higher tendency of low SES students to rely on social support more during adverse or traumatic events. Third, a finding that low parent education students were harmed more than high parent education students by the pandemic in terms of academic, financial, and possibly psychological functioning indicate that resources targeting these areas would reduce the immediate harms that lower SES students are experiencing because of the pandemic.

Lastly, this study found that grit and gratitude before the pandemic predicted better outcomes in terms of harmful impacts that the ensuing pandemic would cause and SWB during the pandemic. Grit was found to contribute to greater pandemic resilience, and both grit and gratitude were found to contribute to increases in SWB during the pandemic for all students, regardless of parental education level. Thus, grit and gratitude intervention would be universally valuable for helping to support and protect the wellbeing of incoming college students during the COVID-19 pandemic. Importantly, the current study also determined that grit and gratitude interventions for college students diverse in SES background are needed. Being more sensitive to the real-life challenges of students would improve intervention. Specifically, grit interventions should involve trained teachers in promoting growth mindset, adaptability and reflection on larger academic goals in students directly in their academic courses (Alan et al., 2019); and gratitude interventions should personalize practices so that they enhance in-person interactions that students have with peers and teachers (Bono et al., 2020; Renshaw \& Hindman, 2017).

\section{Introduction}

Urban universities in the United States have been described as paramount in guaranteeing access to college for students who have been raised in high-needs urban neighborhoods (Martinez-Brawley, 2003). Sometimes referred to as metropolitan universities, these campuses are located in major urban areas across the United States. The student populations of urban universities are often made up of a large population of commuter students and offer undergraduate, graduate and professional programs. The education provided at these 
institutions is created within an urban framework, with research aimed at the local community level (Hill, 1981). This study focuses on students at a medium-large size urban university in a large metropolitan area of the United States. The diversity of student populations in urban universities is ideal for researching how existing inequities are exacerbated in times of crisis. The current study focuses on the stress, social and emotional lives, and resilience of freshmen college students during the beginning months of the COVID-19 pandemic and examines if certain factors helped - specifically, grit and gratitude. The study analyzes participants $(n=86)$ using a shortterm longitudinal study from prior to campus closure on March 10, 2020 to the end of the semester (May 2020), which was completed remotely. In particular, the study examines whether grit and gratitude at the start of semester predicts how much impact the pandemic had on the psychological, academic and financial functioning as well as resilience of students toward the end of the semester.

\section{Literature review}

The university serves a diverse student body and the campus environment benefits from the diversity, but injustice and inequality plague vast segments of America's diverse society. The Stanford Center on Poverty and Inequality (2011) listed 20 different forms of inequality common in America, many of which disproportionally impact urban university students. The current research looked specifically at inequality between low versus high socioeconomic status groups (as determined by parental education level) in terms of the pandemic's psychological, academic and financial impacts as well as resilience to the pandemic.

Stanford reports that racial gaps in education are a form of inequality in the United States (2011). The university population sampled in the current study consists of a majority firstgeneration college students (FGCS) (54.1\%) and a majority Latinx students (60.3\%), with $64.2 \%$ being Pell-eligible. The university is also majority female. Students who have parents with low educational backgrounds, as a population segment, face a wide swath of adjustments that their continuing-generation peers do not experience. These differences impact students not just early in their freshman year of college, but throughout their education (Phillips et al., 2020).

College students also face obstacles to education. A study of poor academic performance in college students $(N=8,997)$ attributed it mostly to stress (Frazier et al., 2019). College students who have experienced Adverse Childhood Experiences (ACEs) face additional educational barriers caused by having to navigate family and health barriers (Hinojosa et al., 2019). Notably, evidence shows that college students tend to use socio-emotional traits more than educational traits when adapting to college life (Gerdes \& Mallinckrodt, 1994). Thus, using qualities like grit and gratitude may prove helpful.

A predominantly female campus population is already predisposed to greater stressdampened academic performance (Frazier et al., 2019). Also, students whose parents have lower education levels are disadvantaged due to experiencing less fit with school (Phillips et al., 2020). Research found that the most common reasons FGCS in a public California university overcame challenges and persisted to graduation were having emotional support from family and friends from home, developing supportive relationships with university faculty, peers, and staff; and believing that college would enable them to achieve future life and career goals (Azmita et al., 2018). These are the very conditions grit and gratitude may help foster. The switch to remote learning during COVID-19 may have left FGCS with additional disadvantages denying them access to these resources.

Immigration and inequality together were listed as a form of inequality in the United States (Stanford, 2011). Nationally, approximately $25 \%$ of the population is either $2^{\text {nd }}$ or $1^{\text {st }}$ generation 
immigrant (Trevelyan et al., 2016). Latinx immigrants who are eligible for Deferred Action for Childhood Arrivals (DACA) already experience stress that their non-DACA peers do not, as the DACA process pushes students into a more adult role sooner (Siemons et al., 2017). A different study identified four domains of stressors that diminish quality of life for university students from immigrant families: parent-child cultural conflict, family disengagement, ethnic discrimination and family financial stress (Bakhtiari et al., 2018). Family financial stress ranked second as most impactful. Importantly, little is known about how grit and gratitude may help such students.

\section{Pandemic Impact}

Early research on the pandemic's effects shows that many aspects of mental health have already been impacted (Bavel, 2020; Stark et al., 2020; Van Agteren et al., 2020; Yang et al., 2020). These impacts have occurred even in homes that do not contain family members who have contracted COVID-19. Thus, the pandemic is a major stressor, even if everyone in the home has remained healthy. Stress can additionally be increased by the forced isolation of quarantine and forced proximity between family members with tenuous relationships (Bavel, 2020; Stark et al., 2020). Additionally, early COVID-19 research suggests that traditional protective factors could be undermined by pandemic stressors (Stark et al., 2020). Some scholars note that renewed ethnocentrism during pandemics can also impact discrimination and prejudice, exacerbating pre-existing feelings of racism against diverse students (Bavel et al., 2020), a phenomenon evident in U.S. society lately.

However, there is hope. The impact of the pandemic may be partially controlled by positive thinking and resilience. Research on college students $(N=384)$ in Wuhan, China found that COVID-19 negatively impacted mental health, but that the effect seems strongly mediated by resilience and positive thinking (Yang et al., 2020). Therefore, grit and gratitude, which are shown to support academic achievement, supportive relationships and resilience (Duckworth, 2007; King \& Datu, 2018; Zheng et al., 2011) may help protect individuals from adversity like the COVID-19 pandemic.

Due to the rarity of global pandemics, there is a literature gap in applicable research. Previous research on the effects of natural disasters and other crises could highlight other possible impacts of COVID-19. Research on the Deepwater Horizon oil spill showed that in addition to death and injuries, natural hazards can cause psychosocial concerns too (Drescher et al., 2014). Additionally, a longitudinal study following Hurricane Katrina survivors found significant physical and mental health consequences: Digestive problems increased seven times, backaches doubled, and migraines or headaches tripled - with women experiencing more of these effects, especially if they had lost a family member (Arcaya et al., 2014).

\section{Grit}

Grit is a personality trait that can be used as a non-cognitive factor in performance and success. Grit is also synonymous with resilience, a quality of passion and determination toward long term goals in the face of challenges and failures (Duckworth et al., 2007). In a 2016 meta-analysis, grit was found to be distinct from, and as supportive as, cognitive abilities for academic success (Credé et al., 2016).

A cross cultural study of grit in college students labelled three distinct characteristics of grit: (1) perseverance of effort, (2) adaptability to situations, and (3) consistency of interest. Of the three domains, perseverance and adaptability were found to positively predict flourishing (Datu et al., 2020). Grit may also predict better academic outcomes. The seminal study on grit found 
that individuals with higher levels of grit have higher academic aptitude scores, complete rigorous training courses and even win spelling bees more frequently (Duckworth, 2007).

\section{Gratitude}

McCullough et al., (2001) describe gratitude as a moral emotion that helps us recognize when others intentionally support our wellbeing. In later work, McCullough and colleagues found support for gratitude as an evolutionary adaptation that not only acknowledges the value of when others benefit our welfare, but that it reinforces others' kindness toward us and spurs mutually cooperative relationships (Forster et al., 2017; Smith et al., 2017). Gratitude is not only an important trait for reinforcing altruistic behavior individually and communally, but it also accounts for reductions in depression, anxiety and substance abuse (Kendler et al., 2003).

Gratitude is also related to feelings of subjective wellbeing (SWB) and may help to build resilience. One study of school age students in China found gratitude to be significantly related to SWB (Tian et al., 2015). Gratitude also may promote protective factors in individuals. Specifically, gratitude not only seems to buffer youth from stress (Reckart et al., 2017), it is associated with building resilience in individuals after major traumas. Gratitude was shown to contribute to reduced PTSD symptoms via resilience (Zheng et al., 2011) and to developing posttraumatic growth (PTG) after the devastating 2008 earthquake in Wenchuan, China (Zhou \& $\mathrm{Wu}, 2015)$.

\section{Subjective Wellbeing among emerging adults and college students}

Research on happiness in university students has found that financial security, academic success and self-esteem can explain most of university students' happiness (Flynn \& MacLeod, 2015), while self-esteem, daily positive events and physical appearance were predictive of higher life satisfaction in a study of Spanish students (Zuffiano et al., 2018). There are, however, obstacles to wellbeing. Wellbeing among Latinx college students is negatively impacted by the experience of microagressions on the college campus (Hernandez \& Villodas, 2020), and Asian students who experienced microaggressions related to feelings of invisibility and sexual stereotypes viewed their campus racial landscape more negatively (Gallo, 2017).

Here too, grit and gratitude may help, especially during adversity. Lin and Kim (2017) found that grit contributed to subjective wellbeing in young adults and that basic needs satisfaction helped account for this. Tian et al. (2016) found this same pattern with gratitude. Further, evidence supports gratitude's protective role during traumatic events. Israeli adolescents traumatized by nearby missile attacks who were more grateful exhibited fewer PTSD symptoms 2.5 months later because of gratitude's links to fewer negative emotions and more life-satisfaction (Israel-Cohen et al., 2015).

\section{Resilience to Pandemic}

Resilience has been shown to possibly be an important trait in mediating the negative mental health impacts of the pandemic on Chinese students in Wuhan (Yang et al., 2020).

Resilience has also had an important role in coping after natural disasters or other crises. The trait, in addition to social support and search for meaning, accounts for significant amounts of variance in the PTG of survivors of the 2016 Louisiana flooding (Boullion et al., 2020).

PTG and substance abuse were also measured among college students in the wake of the 2009 L'Aquila earthquake in Italy, which left over 300 dead, approximately 1,500 injured and over 60,000 residents homeless (Bianchini et al., 2015). The study found evidence of substance use as a negative coping strategy and a strong negative correlation between direct earthquake exposure 
and posttraumatic growth scores. Another recent study measured resilience and PTG of medical students at Fukushima Medical University during the earthquake, tsunami and nuclear disaster in 2011, 8 years post-disaster. Medical students who volunteered in the wake of the incident showed both greater PTG and resilience (Kaye-Kauderer et al., 2019). Additionally, negative feelings such as sadness, anxiety, anger and confusion were positively correlated with resilience.

Studies have shown that those who experience hardship may ultimately become more resilient (Seery et al., 2010). Exposure to adversity also helps to overcome numeracy bias and allows those who have survived hardships to become more compassionate to large groups where there is mass suffering, unlike those who may not have experienced adversity (Lim \& DeSteno, 2019). And coping capacities in college populations have been described as the impetus for students to remain in academic society after having experienced personal trauma (Bulathwatta et al., 2017). Here again, grit and gratitude may help. Both were found to promote resilience, as both helped reduce risk of suicidal ideations by enhancing meaning in life (Kleiman et al., 2013).

\section{Methods}

The current study used a short term longitudinal, correlational design with two time-points. One survey was administered early in the semester and prior to campus closure (January 27 March 10) and another at the end of the semester (April 30 - May 20).

\section{Participants}

Participants for this study $(N=86)$ were undergraduate students at an urban university in the United States with 15,000 students. The average age of participants was 19.38 (SD = 2.48). The study participants were mostly female (81.4\%), and ethnically, mostly Latinx $(79.1 \%)$, followed by African American (14\%), Asian (4.7\%), White (1.2\%) and other (1.2\%). Socioeconomic status (SES) was assessed via parental education. The breakdown for both parents' education was as follows: completed elementary school (11.6\%), middle school (25.6\%), high school (37.2\%), some college $(16.3 \%)$, completed college $(8.1 \%)$, or completed graduate school $(1.2 \%)$. In total, $74.4 \%$ of participants had parents with a high school education or below.

\section{Procedure and Measures}

Participants were freshmen students in Introductory Psychology, who were recruited through the university's human subject pool and participated for course credit. Students provided consent and completed surveys online in a quiet location and time of their choosing. The primary investigator emailed students who completed the first survey the link to the second survey and sent reminders. Six cases with initial surveys after campus closure were dropped, and 2 timepoints were obtained for all participants.

\section{Materials/Surveys}

Table 1 displays the battery of measures that were used in the surveys at T1 and T2. 
Table 1. Measures Used in Surveys at T1 and at T2

\begin{tabular}{|c|c|c|c|}
\hline $\begin{array}{c}\text { Construct } \\
\text { and Citation }\end{array}$ & $\begin{array}{c}\text { Scale Name, Description, Internal Consistency Reliability Statistics and Example/s of } \\
\text { Questions }\end{array}$ & $\begin{array}{l}\text { Use } \\
\text { T1 }\end{array}$ & $\begin{array}{l}\text { Use } \\
\text { T2 }\end{array}$ \\
\hline $\begin{array}{l}\text { Grit } \\
\text { (Duckworth, } \\
2007 \text { ) }\end{array}$ & $\begin{array}{l}\text { The GRIT scale was used to measure grit, which indicates ability to persevere, adapt, and be } \\
\text { consistent and passionate towards the completion of long-term goals. The } 10 \text { items (e.g., "New } \\
\text { ideas and projects sometimes distract me from previous ones") were rated on a } 5 \text {-pt scale (" } 1=\text { Not } \\
\text { at all like me" to " } 5 \text { = Very much like me)" and had acceptable internal consistency }(\alpha=.74) \text {. }\end{array}$ & $X$ & \\
\hline $\begin{array}{l}\text { Grateful } \\
\text { Disposition } \\
\text { and Emotion } \\
\text { (McCullough } \\
\text { et al., 2002) }\end{array}$ & $\begin{array}{l}\text { The Gratitude Questionnaire (GQ-6) was used to assess grateful disposition, which is the tendency } \\
\text { to appreciate more people, places and events/situations strongly and frequently. The } 6 \text { items (e.g., } \\
\text { "I have so much in life to be thankful for") were rated on a 7-pt scale ("1 = Strongly Disagree" to } \\
\text { "7 = Strongly Agree") and showed good reliability ( } \alpha=.82) \text {. } \\
\text { Gratitude Adjective Checklist (GAC) was used to assess grateful emotions/mood. The } 3 \text { items } \\
\text { (frequency of feeling grateful, appreciative and thankful) were rated on a 6-pt scale } \\
\text { ("Never/Almost Never" to "Always/Almost Always") and showed excellent reliability ( } \alpha \mathrm{s}=.93 \\
\text { and } .94 \text { ) respectively. }\end{array}$ & (1) & $X$ \\
\hline $\begin{array}{l}\text { Resilience } \\
\text { (CD-RISC; } \\
\text { Connor \& } \\
\text { Davidson, } \\
2003 \text { ) }\end{array}$ & $\begin{array}{l}\text { The Connor Davidson Resilience Scale was modified to measure resilient responding to the } \\
\text { pandemic. The } 10 \text { items (e.g., "I was able to adapt when changes to my expectations occurred } \\
\text { during COVID-19") were rated on a } 5 \text {-pt scale (" } 1=\text { Disagree" to " } 5=\text { Agree") and showed very } \\
\text { good reliability }(\alpha=.90) \text {. }\end{array}$ & & $X$ \\
\hline $\begin{array}{l}\text { Psychological, } \\
\text { Educational, } \\
\text { and Financial } \\
\text { Impacts of the } \\
\text { Coronavirus } \\
\text { Pandemic } \\
\text { (Conway et } \\
\text { al., 2020). }\end{array}$ & 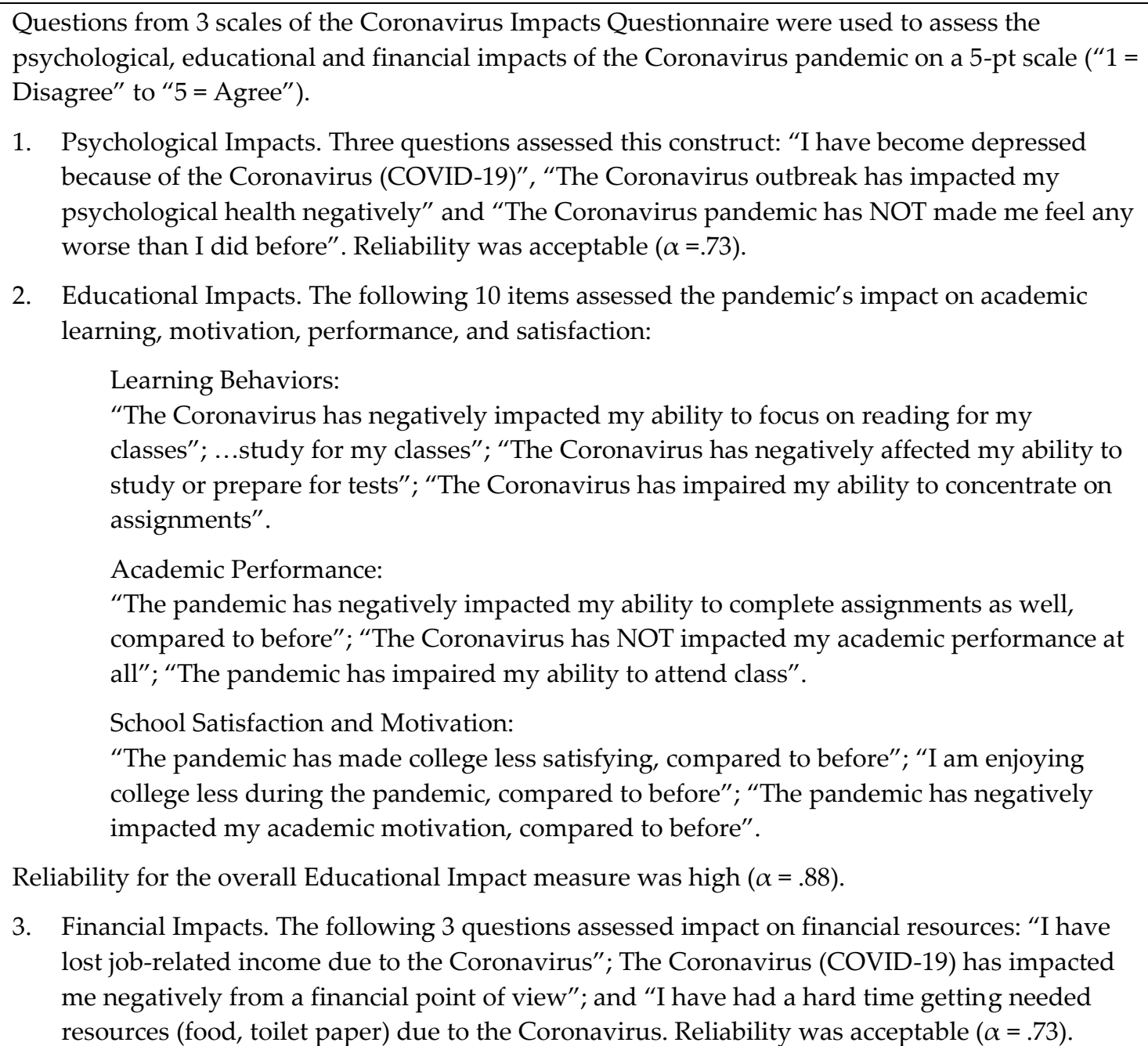 & & $X$ \\
\hline
\end{tabular}




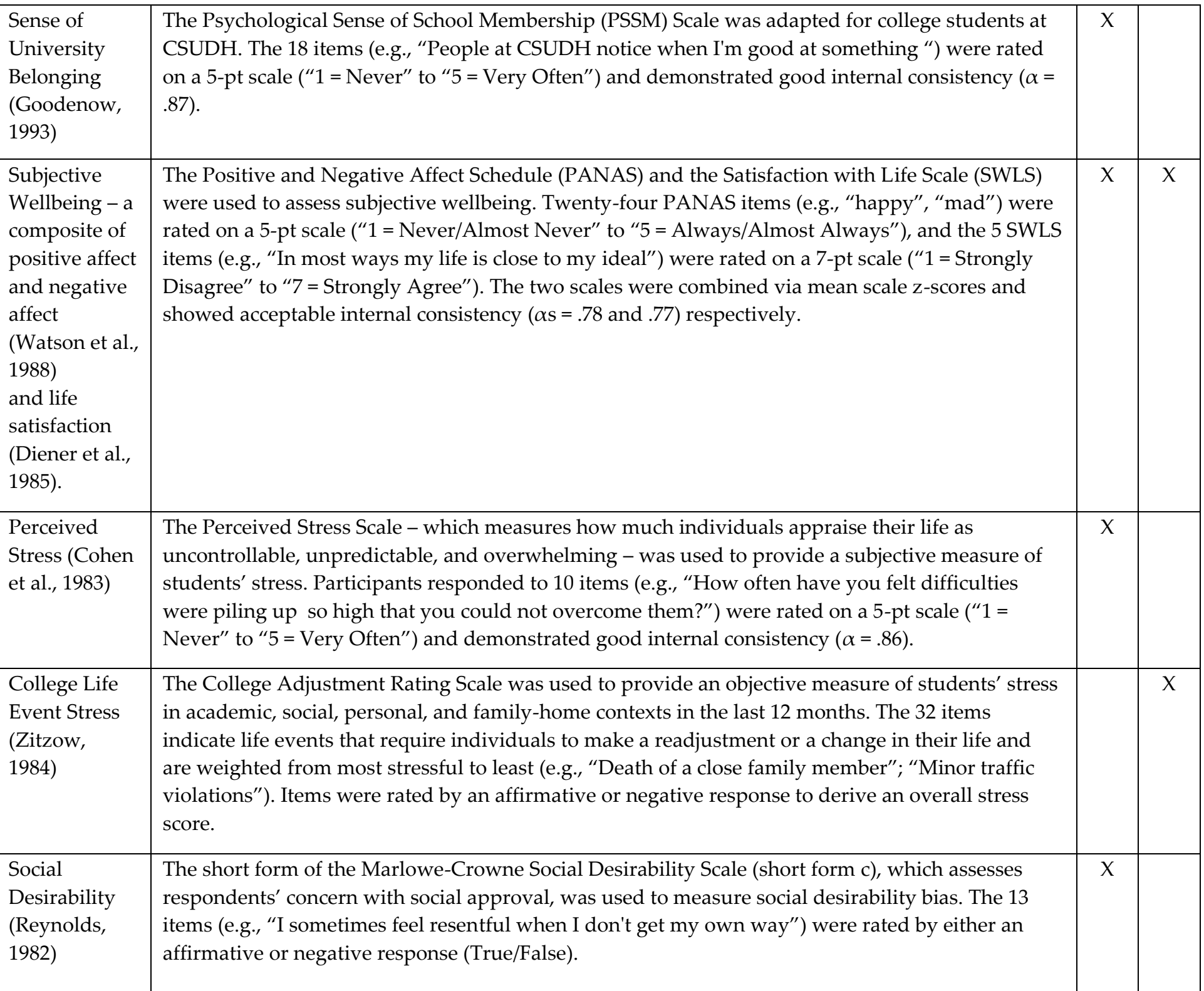

Note: Larger scores indicate greater amounts of the constructs.

\section{Data Analysis}

First, a One-Way ANOVA was conducted to examine differences between low vs. high parental education students in terms of sense of university belonging, grit, trait gratitude, subjective wellbeing (SWB), and perceived stress before the pandemic. Another examined whether there were differences between the two in pandemic impacts on educational, financial and psychological functioning, resilient responding to the pandemic and SWB amidst the pandemic. Table 2 displays descriptive statistics of the main study variables and their correlations at $\mathrm{T} 1$ and $\mathrm{T} 2$. 
Table 2. Descriptive Statistics and Correlations of Main Study Variables.

Time 1 Descriptive Statistics and Bivariate Correlations:

\begin{tabular}{lllllllll}
\hline Variables & & & & University & Perceived & SWB & Grit & Gratitude \\
& $\mathrm{N}$ & Mean & SD & Belonging & Stress & & & \\
\hline University Belonging & 86 & 3.42 & .58 & & $-.464^{* *}$ & $.400^{* *}$ & $.263^{*}$ & $.384^{* *}$ \\
Perceived Stress & 86 & 3.13 & .65 & & & $-.475^{* *}$ & $-.505^{* *}$ & $-.511^{* *}$ \\
SWB & 86 & 3.93 & .55 & & & & $.362^{* *}$ & $.572^{* *}$ \\
Grit & 86 & 3.35 & 0.53 & & & & & $.431^{* *}$ \\
Gratitude & 86 & 5.71 & 1.00 & & & & & \\
\hline
\end{tabular}

${ }^{*} p<.05 ;{ }^{* *} p<.01$

Time 2 Descriptive Statistics:

\begin{tabular}{llll}
\hline \multicolumn{1}{c}{ Variable } & $\mathrm{N}$ & Mean & SD \\
\hline Life Event Stress Score & 86 & 298.62 & 179.45 \\
Pandemic Financial Impact & 86 & 3.01 & 1.20 \\
Pandemic Psychological Impact & 86 & 2.99 & 1.15 \\
Pandemic Educational Impact & 86 & 3.96 & .86 \\
Pandemic Resilience & 86 & 3.51 & .85 \\
Change in Grateful Mood & 86 & .05 & .70 \\
Parents' Educational Level & 86 & 3.35 & .53 \\
SWB (z-score) & 86 & .00 & .90 \\
\hline
\end{tabular}

Time 2 Bivariate Correlations:

\begin{tabular}{llllllll}
\hline & $\begin{array}{l}\text { Pandemic } \\
\text { FI }\end{array}$ & $\begin{array}{l}\text { Pandemic } \\
\text { PI }\end{array}$ & $\begin{array}{l}\text { Pandemic } \\
\text { AI }\end{array}$ & $\begin{array}{l}\text { Pandemic } \\
\text { Resilience }\end{array}$ & $\begin{array}{l}\text { Grateful } \\
\Delta\end{array}$ & $\begin{array}{l}\text { Life } \\
\text { Stress }\end{array}$ & $\begin{array}{l}\text { Parents } \\
\text { EDU }\end{array}$ \\
\hline Pandemic PI & $.284^{* *}$ & & & & & & \\
Pandemic AI & $.331^{* *}$ & $.307^{* *}$ & & & & & \\
P. Resilience & $-.276^{*}$ & $-.436^{* *}$ & $-.499^{* *}$ & & & & \\
Grateful $\Delta$ & -.122 & -.081 & .033 & .159 & & & \\
Life Stress & $.266^{*}$ & $.233^{*}$ & $.431^{* *}$ & -.212 & -.035 & & \\
Parents EDU & $-.254^{*}$ & -.089 & -.185 & .133 & -.157 & -.054 & \\
SWB & $-.258^{*}$ & $-.367^{* *}$ & -.132 & $.454^{* *}$ & .066 & -.139 & $.214^{*}$ \\
\hline
\end{tabular}

${ }^{*} p<.05 ;{ }^{* *} p<.01$

Note: $F I=$ Financial Impact, $P I=$ Psychological Impact, $A I=$ Academic Impact, Grateful $\Delta=$ change in grateful emotions T1-T2,

Life Stress $=$ Life Events Stress Score

Next, we examined whether grit and gratitude early in the semester (pre-pandemic) each predicted the impact that the pandemic had on psychological and educational functioning and on resilient responding to the pandemic at the end of the semester in separate hierarchical multiple linear regressions, controlling for sex, life event stress, and social desirability. We then repeated these analyses with an additional covariate for parent education to see if results held above and beyond the effects of SES. Finally, we conducted similar regression analyses to examine if grit and gratitude each predicted increases in SWB during the pandemic by additionally including a covariate of T1 SWB. 


\section{Results and Discussion}

The first ANOVA results showed there were significant differences between students of high versus low parental education in terms of perceived stress, $F(1,84)=5.97, p=.017$, SWB,$F(1,84)$ $=6.14, p=.015$, and trait gratitude, $F(1,84)=8.05, p=.006$. See top of Table 3 . The low group reported greater perceived stress than the high group, $M=3.25(S D=0.63)$ vs. $M=2.91(S D=$ $0.63)$, less $S W B, M=3.81(S D=0.58)$ vs. $M=4.13(S D=0.44)$, and less trait gratitude, $M=5.49(S D$ $=1.07)$ vs. $M=6.08(S D=0.68)$. Differences in university belonging were not significant, which is not surprising given the robust climate of support for the academic success of minority and $1^{\text {st }}$ generation college students at the urban university where this study occurred.

The second ANOVA revealed significant differences between students of high versus low parental education in terms of COVID-19 financial impact, $F(1,84)=5.13, p=.026$ and COVID-19 academic impact, $F(1,84)=4.32, p=.041$. There was a positive marginal effect on psychological impact too, $F(1,84)=2.97, p=.089$. See middle of Table 3. The low group reported more financial impact than the high group, $M=3.23(S D=1.06)$ vs. $M=2.64(S D=1.34)$, more academic impact, $M=4.11(S D=0.76)$ vs. $M=3.72(S D=0.97)$, and possibly more psychological impact, $M=3.15$ $(S D=1.02)$ vs. $M=2.72(S D=1.31)$. No significant differences emerged in pandemic resilience and, unexpectedly, life event stress.

Given the fact that we found significant differences in trait gratitude at T1 between students of high vs. low parental education, we explored if levels of gratitude between the two groups remained different at $\mathrm{T} 2$ too or if they fluctuated. Thus, we conducted an additional ANOVA on grateful emotions at T1, T2, and on T1-T2 change. This additional analysis showed that while the low group experienced fewer grateful emotions at $\mathrm{T} 1(M=4.62, S D=1.07)$ than the high group $(M=5.16, S D=0.87), F(1,84)=5.72, p=.019$, there were no differences at T2. More importantly, group differences emerged in change in grateful emotions from T1 to T2, $F(1,84)=4.83, p=.031$ (see bottom of Table 3). Whereas the low group increased in grateful emotions during the pandemic $(M=0.18, S D=0.70)$, the high group decreased $(M=-0.16, S D=0.66)$.

Table 3. ANOVA Effects of Low Vs. High Parent Education Students on Dependent Variables at $\mathrm{T} 1, \mathrm{~T} 2$.

Time 1 ANOVAs: Effects on University Belonging, Perceived Stress, Subjective Wellbeing, and Gratitude

\begin{tabular}{llccccc}
\hline & & Sum of & & Mean & & \\
& & Squares & df & Square & F & Sig. \\
\hline University & Between Groups & .02 & 1 & .02 & .07 & .791 \\
Belonging & Within Groups & 28.38 & 84 & .34 & & \\
& Total & 28.40 & 85 & & & \\
\hline Perceived Stress & Between Groups & 2.38 & 1 & 2.38 & 5.97 & .017 \\
& Within Groups & 33.47 & 84 & .40 & & \\
& Total & 35.85 & 85 & & & \\
\hline \multirow{2}{*}{ Subjective wellbeing } & Between Groups & 4.75 & 1 & 4.75 & 6.14 & .015 \\
& Within Groups & 64.999 & 84 & .77 & & \\
& Total & 69.747 & 85 & & & \\
\hline \multirow{2}{*}{ GQ6 } & Between Groups & 7.13 & 1 & 7.13 & 8.05 & .006 \\
& Within Groups & 74.381 & 84 & .89 & & \\
& Total & 81.510 & 85 & & & \\
\hline
\end{tabular}


Time 2 ANOVAs: Effects on Life Event Stress, Pandemic Impacts (Financial, Psychological, Academic), Pandemic Resilience, and T1-T2 Change in Grateful Emotions.

\begin{tabular}{|c|c|c|c|c|c|c|}
\hline & & $\begin{array}{l}\text { Sum of } \\
\text { Squares }\end{array}$ & $\mathrm{df}$ & $\begin{array}{l}\text { Mean } \\
\text { Square }\end{array}$ & $\mathrm{F}$ & Sig. \\
\hline \multirow[t]{3}{*}{ Life Event Stress } & Between Groups & 442.370 & 1 & 442.37 & .01 & .908 \\
\hline & Within Groups & 2736729.968 & 84 & 32580.12 & & \\
\hline & Total & 2737172.337 & 85 & & & \\
\hline Pandemic & Between Groups & 7.065 & 1 & 7.07 & 5.13 & .026 \\
\hline \multirow[t]{2}{*}{ Financial Impact } & Within Groups & 115.596 & 84 & 1.38 & & \\
\hline & Total & 122.661 & 85 & & & \\
\hline Pandemic & Between Groups & 3.812 & 1 & 3.81 & 2.97 & .089 \\
\hline Psychological & Within Groups & 107.961 & 84 & 1.29 & & \\
\hline Impact & Total & 111.773 & 85 & & & \\
\hline Pandemic & Between Groups & 3.048 & 1 & 3.05 & 4.32 & .041 \\
\hline \multirow[t]{2}{*}{ Academic Impact } & Within Groups & 59.314 & 84 & .71 & & \\
\hline & Total & 62.362 & 85 & & & \\
\hline Pandemic & Between Groups & .439 & 1 & .44 & .60 & .441 \\
\hline \multirow[t]{2}{*}{ Resilience } & Within Groups & 61.501 & 84 & .73 & & \\
\hline & Total & 61.941 & 85 & & & \\
\hline Grateful Emotion & Between Groups & 2.258 & 1 & 2.26 & 4.83 & .031 \\
\hline \multirow[t]{2}{*}{ Change T1-T2 } & Within Groups & 39.266 & 84 & .47 & & \\
\hline & Total & 41.525 & 85 & & & \\
\hline
\end{tabular}

Note: Grateful Emotion, unlike the other T2 dependent measures, was calculated as a difference score.

Multiple linear regression analyses revealed that grit and gratitude at T1 were predictive of positive effects at T2, above and beyond differences in sex, life event stress, and social desirability. Grit significantly predicted greater pandemic resilience, $t=2.24, p=.028, \Delta R^{2}=.055$, increases in SWB, $t=5.07, p=.000, \Delta R^{2}=.328$, and marginally lower psychological and academic impacts. See Table 4. The standardized regression coefficients relating grit to pandemic resilience, psychological harm, and increases in SWB, were medium in size $(\beta s=.246,-.213, .483)$ and small for academic impact $(\beta=-.172)$. Therefore, students with more grit demonstrated higher levels of pandemic resilience, increases in SWB, and perhaps lower psychological and academic harms due to the pandemic. Controlling additionally for parental education, however, grit only significantly predicted pandemic resilience, $t=2.07, p=.042, \Delta R^{2}=.050$, and increases in $\mathrm{SWB}, t=4.73, p=.000, \Delta R^{2}=.333$. The standardized regression coefficients relating grit to pandemic resilience and increases in SWB shrank but remained medium in size in these models $(\beta s=.235, .465)$.

The regression models analysing whether trait gratitude predicted pandemic outcomes showed that T1 trait gratitude predicted less psychological harm during COVID-19, $t=-2.26, p=$ .026 , increases in SWB $, t=6.95, p=.000, \Delta R^{2}=.445$, and marginally greater pandemic resilience. The standardized regression coefficients relating gratitude to psychological harm and SWB were medium in size $(\beta=-.239, .584)$ and small for pandemic resilience $(\beta=.188)$. Therefore, students with more trait gratitude experienced less psychological impact, increases in SWB during the pandemic and possibly greater resilience. Controlling additionally for parental education, however, gratitude only significantly predicted SWB, $t=6.57, p=.000, \Delta R^{2}=.446$. The 
standardized regression coefficient relating gratitude to increases in SWB remained medium in size $(\beta=.590)$.

Table 4. (Regression) Multiple Linear Regressions of Grit and Gratitude on Dependent Variables.

\begin{tabular}{llcccc}
\hline Predictor Variable & Dependent Variable & \multicolumn{1}{c}{$\beta$} & \multicolumn{1}{c}{$\Delta$} & $\Delta R^{2}$ & $p$ \\
\hline Grit & Pandemic Edu Impact & -.172 & -1.72 & .027 & .089 \\
& Pandemic Psy Impact & -.213 & -1.95 & .041 & .055 \\
& Pandemic Resilience & .246 & 2.24 & .055 & $.028^{*}$ \\
& SWB & .483 & 5.07 & .328 & $.000^{* *}$ \\
Gratitude & & & & \\
& Pandemic Edu Impact & .023 & .232 & .001 & .817 \\
& Pandemic Psy Impact & -.239 & -2.26 & .055 & $.026^{*}$ \\
& Pandemic Resilience & .188 & 1.74 & .034 & .085 \\
& SWB $^{1}$ & .584 & 6.95 & .445 & $.000^{* *}$ \\
\hline
\end{tabular}

${ }^{*} p<.05 ;{ }^{* *} p<.001 ;{ }^{1}$ Analyses with Subjective Wellbeing (SWB) included an additional covariate for SWB at T1 (prior to the pandemic).

\section{The Path Forward}

The results of this study find that trait gratitude and grit may be especially beneficial for students from low socioeconomic backgrounds, specifically FGCS and others from homes with low parental education levels. Luckily, overall research shows both grit and gratitude to be modifiable, which indicates that they are therefore useful as part of therapeutic interventions. The impact of urban universities on students with low parental education cannot be overstated. These institutions are designed to help those from high need urban areas, many of whom are first generation college students. The university where this study occurred is such an institution. Though sparse, some recent research does point to promising grit and gratitude frameworks and interventions for urban college students.

As previously mentioned, grit is comprised of 3 components: adaptability to situations, consistency of interest, and perseverance of effort. Cross cultural research found that adaptability and perseverance predicted flourishing among Polish, Filipino, and Japanese undergraduate students (Datu et al., 2020). Thus, specifically targeted interventions aimed at these components may increase grit. One specific intervention aimed at persistence in postsecondary STEM programs by FGCS found that increased persistence was correlated with positive impacts on performance (Hecht et al., 2019). Using a utility-value intervention, this study required students to journal about the personal value of STEM classes specific to their major and measured persistence behaviorally through continued course-taking and remaining in the biomed program after two years. The possible benefit of a program like this is that it can easily be adapted to other majors and programs and can also be employed remotely, which is valuable given the fact that the current pandemic situation remains tumultuous.

The most promising grit interventions specifically for lower SES college students may involve promoting growth mindset in some way. Though a recent meta-analysis concluded that the overall effects of such interventions are weak and may only benefit low SES students who are low-achieving or academically at risk (Sisk et al., 2018), one factor that appears to help is having trained teachers deliver such interventions themselves. For example, one such study examined a randomized intervention with low SES elementary students in Turkey that instilled optimistic beliefs about the productivity of effort and encouraged students to persevere through setbacks (Alan et al., 2019). The intervention involved a teacher-training program that focused on 3 interrelated ideas underlying grit: growth mindset, perseverance through failures, and goal 
setting. These researchers found significant effects on an incentivized real-effort task and performance in standardized math tests 2.5 years later. But such a program has not been tested in college settings.

In the face of changing university structure during the COVID-19 pandemic, online accessible interventions aimed at helping students address stress, depression, and wellbeing, may also provide a way forward. Research has shown that intention to use online interventions increases as students experience higher levels of distress (Ryan et al., 2010). Online interventions are also accessible globally and the current generation of college students is tech savvy enough to operate online interventions (Palma-Gómez et al., 2020).

A recent quasi-experimental study by Bono et al. (2020) found that an intervention combining a research-based curriculum and a thanking/journaling app was effective with predominantly Latinx students in lower-SES urban high schools. They found that the intervention increased subjective wellbeing and friendship satisfaction and decreased stress and anxiety and depression symptoms in students. Such an intervention, that makes use of an app for students to express thanks to peers and teachers and to genuinely practice gratitude, has not been used with college students, but such techniques could be adapted to help personalize and enhance online learning in college too - especially in courses that involve collaborative learning, where social support and thanks can be exchanged naturally.

Finally, one study similarly used a technology-based gratitude intervention with college students (Renshaw \& Hindman, 2017). Employing a randomized controlled design, these researchers adapted the gratitude letter-writing-and-visit method by using notes instead of letters and instant communication technology instead of personal visits. Results indicated that, compared to active control conditions, the intervention had greater positive effects on optimism and school connectedness, but that it also had small effects on depression and gratitude. Future research should examine if such gratitude interventions are effective with college students who are diverse in terms of SES.

Moving forward, research with student populations in urban universities will be key. Such research can help better address racial and SES gaps in education as well as the additional obstacles due to immigration and other inequities experienced by urban university students. It can also examine whether grit and gratitude moderate the impacts of such obstacles on students' academic functioning, mental health and wellbeing. Future research recommendations should close the literature gap on grit and gratitude traits in urban university students to pave the way for more tailored interventions.

\section{Conclusion}

The current study examined whether socioeconomic status (parental education) was related with perceived stress, SWB, grit and gratitude prior to the pandemic, and with COVID-19 impacts (psychological, academic and financial wellbeing) and pandemic resilience in a sample of urban university students. Researchers were also interested in whether inequities in education due to socioeconomic disadvantages, as experienced by many FGCS or urban college students, might be exacerbated by conditions caused by the pandemic. Additionally, it explored if grit and gratitude could serve as coping strategies for alleviating these pandemic impacts and improving pandemic resilience and SWB overall. The investigators were fortunate to collect baseline data prior to the onset of COVID-19. It was hypothesized that lower parental education would negatively impact stress and SWB and exacerbate pandemic outcomes and that grit and gratitude would positively influence SWB and pandemic outcomes. Hypotheses were largely supported. 


\section{Parental Education Level}

We found that students with lower parent education (i.e., high school or less) reported more perceived stress, less SWB, and less gratitude before the pandemic, compared to students with higher parent education (undergraduate college or higher). They also experienced worse academic and financial impacts due to the pandemic, compared to their higher-parent education peers - a pattern consistent with the disproportionate consequences plaguing U.S. minorities during the COVID-19 pandemic.

Grit

The findings of this study suggest, surprisingly, that the relationship between grit and academic functioning was not statistically significant. Yet, in the current sample, grit was significantly associated with greater pandemic resilience, increases in SWB and, possibly, decreased psychological harm. This suggests that interventions increasing grit characteristics perseverance, adaptability, and consistency - help improve university students' psychological wellbeing during the COVID-19 pandemic and resilience to it.

\section{Gratitude}

Exploring state and trait gratitude, the data from the current sample suggested that trait gratitude was not a factor influencing the pandemic's harmful academic impact. However, it was significantly associated with decreased psychological harm, increased SWB and, possibly, greater resilience during COVID-19. Furthermore, grateful mood increased from before to during the pandemic for lower SES students, whereas the opposite was observed for higher SES students. Therefore, it may be more challenging to promote gratitude in lower-SES students in general, presumably because of greater daily stress, but they may be prone to using gratitude to cope with the adversity of the pandemic more than their higher-SES peers. Urban universities are suitable places for research examining why promoting gratitude in low SES students is more challenging and how to create impactful interventions aimed at those students.

\section{Practical Guidance}

The literature review from this study highlights that urban university students who are FGCS or come from households with low parental education may already be facing an uphill battle (Azmitia et al., 2018; Phillips et al., 2020), even in times without social upheaval or international crisis. The data from this study supports the conclusion that college students who are FGCS or come from homes with low parental education are not faring as well as their later generation peers. The impacts to this vulnerable population are both academic and financial, exacerbating already fragile dynamics.

The population of students where this study occurred is $54 \%$ FGCS. It is reasonable to infer from our results that many, if not majorities, of students from such urban universities are experiencing worse financial and academic outcomes due to the COVID-19 pandemic. Study respondents mostly came from low education backgrounds, with $74.4 \%$ having parents who had completed high school or less (this includes $25.6 \%$ having parents with education up through middle school and $11.6 \%$ with education up through elementary school). Thus, such exacerbations in quality of life due to the pandemic may be occurring in urban college students throughout the United States, putting these important institutions at risk of being unable to adequately support the academic development of many of their students. Urban universities are vital resources for the social mobility of students from high-need backgrounds and the local communities that these institutions serve (Hill, 1981; Martinez-Brawley, 2003). Therefore, long- 
term and broader repercussions could be expected due to the disproportionate hardship many urban college students are experiencing during COVID-19.

Results from this study also support possible future frameworks for interventions that may help this population gain coping strategies aimed at pandemic resilience. Increased pandemic resilience for FGCS and socioeconomically disadvantaged college students would help to alleviate the variance in educational and financial impacts such students are experiencing during the COVID-19 pandemic. The goal of these interventions to increase pandemic resilience would be to raise the grit and gratitude levels of students from backgrounds with low parental education. The lack of grit interventions aimed specifically at FGCS and those from low socioeconomic backgrounds is part of the problem, in addition to the need for more research on how to best promote gratitude in urban university populations.

The university where this study took place is like many urban universities and hosts a variety of terminal degree and credential programs aimed at creating a pathway for students from the beginning of their undergraduate degree into a career-path. Many of these careers have been deemed as essential workers during the COVID-19 pandemic. Of the credential and graduate degrees offered at the university: $28.5 \%$ of graduate students are getting teaching credentials, $14.5 \%$ an MA in education, $13.7 \%$ an MS in nursing, $6.7 \%$ MS in in occupational therapy, $4.7 \%$ an MSW program and $4.2 \%$ in an MFT program. As of the publication of this study (mid-August) the United States has had 5,064,171 cases of COVID-19 (with a 7-day average of daily increases of 52,193 cases) and 162,407 total deaths (CDC, 2020). The future professionals in these muchneeded career fields for a post-pandemic world are currently the undergraduate students in this study and in urban universities across the United States. Not only would they greatly benefit from targeted interventions promoting grit and gratitude, so would society, now and in a postpandemic world.

\section{Authors}

Giacomo Bono

California State University Dominguez Hills

Gbono@csudh.edu

Kresimir Reil

California State University Dominguez Hills

Jadwiga Hescox

California State University Dominguez Hills

\section{Publishing Timeline}

Received 7 July 2020

Accepted 3 August 2020

Published 13 August 2020

\section{References}

Alan, S., Boneva, T., \& Ertac, S. (2019). Ever failed, try again, succeed better: Results from a randomized educational intervention on grit. The Quarterly Journal of Economics, 134(3), 1121-1162. doi:10.1093/qje/qjz006.

Anh, M., \& Davis, H. (2019). Four domains of students' sense of belonging to university. Studies in Higher Education, 45(3), 622-634. 
Arcaya, M. C., Lowe, S. R., Rhodes, J. E., Waters, M. C., \& Subramanian, S. V. (2014). Association of PTSD symptoms with asthma attacks among hurricane Katrina survivors. Journal of Traumatic Stress, 27(6), 725-729. doi: 10.1002/jts.21976.

Azmitia, M., Sumabat-Estrada, G., Cheong, Y., \& Covarrubias, R. (2018). “Dropping out is not an option": How educationally resilient first-generation students see the future. New Directions for Child and Adolescent Development, 2018(160), 89-10010.1002/cad.20240

Bakhtiari, F., Benner, A. D., \& Plunkett, S. W. (2018). Life quality of university students from immigrant families in the United States. Family and Consumer Sciences Research Journal, 46(4), 331-346. DOI:10.1111/fcsr.12260

Bavel, J.J.V., Baicker, K., Boggio, P.S. et al. (2020). Using social and behavioural science to support COVID-19 pandemic response. Nature Human Behaviour, 4, 460-471. https://doi.org/10.1038/s41562020-0884-Z

Bianchini, V., Roncone, R., Giusti, L., Casacchia, M., Cifone, M. G., \& Pollice, R. (2015). PTSD growth and substance abuse among a college student community: Coping strategies after 2009 L'Aquila earthquake. Clinical Practice and Epidemiology in Mental Health, 11, 140-143. https://doi.org/10.2174/1745017901511010140

Bono, G., Mangan, S., Fauteux, M., \& Sender, J. (2020). Effective gratitude interventions in schools for supporting student mental health and wellbeing. The Journal of Positive Psychology. https://doi.org/10.1080/17439760.2020.1789712

Boullion, G. Q., Pavlacic, J. M., Schulenberg, S. E., Buchanan, E. M., \& Steger, M. F. (2020). Meaning, social support, and resilience as predictors of posttraumatic growth: A study of the Louisiana flooding of August 2016. The American Journal of Orthopsychiatry. https://doi.apa.org/doi/10.1037/ort0000464

Bulathwatta, A. D. N., Witruk, E., \& Reschke, K. (2017). Effect of emotional intelligence and resilience on trauma coping among university students. Health Psychology Report, 5(1), 12-19. https://doi:10.5114/hpr.2017.61786

CDC. (2020). Coronavirus disease 2019. https://www.cdc.gov/coronavirus/2019-ncov/casesupdates/cases-in-us.html

Champlin, S., \& Nisbett, G. (2018). Promoting mental health resource use on campus by “trying something new." American Journal of Health Promotion, 32(4), 1140-1144. https://doi.org/10.1177\%2F0890117117740348

Credé M, Tynan MC, Harms PD. (2017). Much ado about grit: A meta-analytic synthesis of the grit literature. Journal of Personality and Social Psychology, 113(3), 492-511. https://doi:10.1037/pspp0000102

Datu, J. A. D., McInerney, D. M., Żemojtel-Piotrowska, M., Hitokoto, H., \& Datu, N. D. (2020). Is Grittiness Next to Happiness? Examining the Association of Triarchic Model of Grit Dimensions with Well-Being Outcomes. Journal of Happiness Studies, 1-29. https://doi.org/10.1007/s10902-02000260-6

Drescher, C. F., Schulenberg, S. E., \& Smith, C. V. (2014). The Deepwater Horizon oil spill and the Mississippi Gulf Coast: Mental health in the context of a technological disaster. American Journal of Orthopsychiatry, 84, 142-151. http://dx.doi.org/10.1037/h0099382

Duckworth, A. L., Peterson, C., Matthews, M. D., \& Kelly, D. R. (2007). Grit: Perseverance and passion for long term goals. Journal of Personality and Social Psychology, 92(6), 1087-1101. https://doi:10.1037/0022$\underline{3514.92 .6 .1087}$ 
First, J., First, N. L., \& Houston, J. B. (2018). Resilience and Coping Intervention (RCI): A group intervention to foster college student resilience. Social Work with Groups, 41(3), 198-210. https://doi.org/10.1080/01609513.2016.1272032

Flynn, D. M., \& MacLeod, S. (2015). Determinants of happiness in undergraduate university students. College Student Journal, 49(3), 452-460.

Forster, D. E., Pedersen, E. J., Smith, A., McCullough, M. E., \& Lieberman, D. (2017). Benefit valuation predicts gratitude. Evolution and Human Behavior, 38(1), 18-26.

http://dx.doi.org/10.1016/j.evolhumbehav.2016.06.003

Frazier, P., Gabriel, A., Merians, A., \& Lust, K. (2019). Understanding stress as an impediment to academic performance. Journal of American College Health, 67(6), 562-570. https://doi.org/10.1080/07448481.2018.1499649

Freeman, T., Anderman, L., \& Jensen, J. (2007). Sense of Belonging in College Freshmen at the Classroom and Campus Levels. The Journal of Experimental Education, 75(3), 203-220. Retrieved June 29, 2020, from www.jstor.org/stable/20157456

Gallo, D. J. (2017). The impact of racial microaggressions on Asian American college students' satisfaction with social support, sense of belonging, and campus racial climate [ProQuest Information \& Learning]. In Dissertation Abstracts International: Section B: The Sciences and Engineering (Vol. 77, Issue 8-B(E)).

Gerdes, H., \& Mallinckrodt, B. (1994). Emotional, social, and academic adjustment of college students: a longitudinal study of retention. Journal of Counseling $\mathcal{E}$ Development, 72, 281-288. DOI:10.1002/j.15566676.1994.tb00935.x

Goodenow, C. (1993). The psychological sense of school membership among adolescents: Scale development and educational correlates. Psychology in the Schools, 30(1), 79-90.

Hecht, C. A., Harackiewicz, J. M., Priniski, S. J., Canning, E. A., Tibbetts, Y., \& Hyde, J. S. (2019).

Promoting persistence in the biological and medical sciences: An expectancy-value approach to intervention. Journal of Educational Psychology, 111(8), 1462-1477.

http://dx.doi.org/10.1037/edu0000356

Hill, S.R. (1981). Urban universities. Twentieth century phenomena. Phi Kappa Phi Journal National Forum, 61(3), 38-39.

Hinojosa, R., Nguyen, J., Sellers, K., \& Elassar, H. (2019). Barriers to college success among students that experienced adverse childhood events. Journal of American College Health, 67(6), 531-540. https://doi.org/10.1080/07448481.2018.1498851

Jin, B., \& Kim, J. (2017). Grit, basic needs satisfaction, and subjective well-being. Journal of Individual Differences, 38(1), 29-35. https://doi.org/10.1027/1614-0001/a000219

Kaye-Kauderer, H. P., Levine, J., Takeguchi, Y., Machida, M., Sekine, H., Taku, K., Yanagisawa, R., \& Katz, C. (2019). Post-traumatic growth and resilience among medical students after the March 2011 disaster in Fukushima, Japan. The Psychiatric Quarterly, 90(3), 507-518. https://doi.org/10.1007/s11126-019-09646-z

Kendler, K. S., Liu, X., Gardner, C. O., McCullough, M. E., Larson, D., \& Prescott, C. A. (2003). Dimensions of religiosity and their relationship to lifetime psychiatric and substance use disorders. American Journal of Psychiatry, 160, 496-503. https://doi.org/10.1176/appi.ajp.160.3.496

King, R. B., \& Datu, J. A. D. (2018). Grateful students are motivated, engaged, and successful in school: Cross-sectional, longitudinal, and experimental evidence. Journal of School Psychology, 70, 105-122. https://doi.org/10.1016/j.jsp.2018.08.001 
Kleiman, E. M., Adams, L. M., Kashdan, T. B., \& Riskind, J. H. (2013). Gratitude and grit indirectly reduce risk of suicidal ideations by enhancing meaning in life: Evidence for a mediated moderation model. Journal of Research in Personality, 47(5), 539-546. http://dx.doi.org/10.1016/j.jrp.2013.04.007

Koydemir, S., \& Sun-Selışık, Z. E. (2016). Well-being on campus: Testing the effectiveness of an online strengths-based intervention for first year college students. British Journal of Guidance $\mathcal{E}$ Counselling, 44(4), 434-446. https://doi.org/10.1080/03069885.2015.1110562

Lambert, L., Passmore, H.-A., \& Joshanloo, M. (2019). A positive psychology intervention program in a culturally-diverse university: Boosting happiness and reducing fear. Journal of Happiness Studies: An Interdisciplinary Forum on Subjective Well-Being, 20(4), 1141-1162. https://doi.org/10.1007/s10902-0189993-Z

Lim, D., \& DeSteno, D. (2019, August 15). Past Adversity Protects Against the Numeracy Bias in Compassion. Emotion. Advance online publication.

Martinez-Brawley, E. (2003). The metropolitan mission of a research university: A study of the context and opportunities for faculty. Arizona State University.

Palma-Gómez, A., Herrero, R., Baños, R., García-Palacios, A., Castañeiras, C., Fernandez, G. L., Llull, D. M., Torres, L. C., Barranco, L. A., Cárdenas-Gómez, L., \& Botella, C. (2020). Efficacy of a self-applied online program to promote resilience and coping skills in university students in four Spanishspeaking countries: Study protocol for a randomized controlled trial. BMC Psychiatry, 20. https://doi.org/10.1186/s12888-020-02536-w

Phillips, L. T., Stephens, N. M., Townsend, S. S. M., \& Goudeau, S. (2020). Access is not enough: Cultural mismatch persists to limit first-generation students' opportunities for achievement throughout college. Journal of Personality and Social Psychology. http://dx.doi.org/10.1037/pspi0000234

Reckart, H., Huebner, E. S., Hills, K. J., \& Valois, R. F. (2017). A preliminary study of the origins of early adolescents' gratitude differences. Personality and Individual Differences, 116, 44-50.

http://dx.doi.org/10.1016/j.paid.2017.04.020

Renshaw, T. L., \& Hindman, M. L. (2017). Expressing gratitude via instant communication technology: A randomized controlled trial targeting college students' mental health. Mental Health E Prevention, 7, 37-44. https://doi.org/10.1016/j.mhp.2017.08.001

Ryan, M. L., Shochet, I. M., \& Stallman, H. M. (2010). Universal online interventions might engage psychologically distressed university students who are unlikely to seek formal help. Advances in Mental Health, 9(1), 73-83. https://doi.org/10.5172/jamh.9.1.73

Seery, M. D., Holman, E. A., \& Silver, R. C. (2010). Whatever does not kill us: Cumulative lifetime adversity, vulnerability, and resilience. Journal of Personality and Social Psychology, 99, 1025-1041. http://dx.doi.org/10.1037/a0021344 2.

Siemons, R., Raymond-Flesch, M., Auerswald, C. L., \& Brindis, C. D. (2017). Coming of age on the margins: Mental health and wellbeing among Latino immigrant young adults eligible for Deferred Action for Childhood Arrivals (DACA). Journal of Immigrant and Minority Health, 19(3), 543-551. DOI 10.1007/s10903-016-0354-x

Sisk, V. F., Burgoyne, A. P., Sun, J., Butler, J. L., \& Macnamara, B. N. (2018). To what extent and under which circumstances are growth mind-sets important to academic achievement? Two metaanalyses. Psychological Science, 29(4), 549-571. https://doi.org/10.1177/0956797617739704

Smith, A., Pedersen, E. J., Forster, D. E., McCullough, M. E., \& Lieberman, D. (2017). Cooperation: The roles of interpersonal value and gratitude. Evolution and Human Behavior, 38(6), 695-703.

http://dx.doi.org/10.1016/j.evolhumbehav.2017.08.003 
Stanford Center on Poverty and Inequality. (2011). 20 facts about U.S. inequality that everyone should know. https://inequality.stanford.edu/publications/20-facts-about-us-inequality-everyone-shouldknow

Stark, A. M., White, A. E., Rotter, N. S., \& Basu, A. (2020). Shifting from survival to supporting resilience in children and families in the COVID-19 pandemic: Lessons for informing US mental health priorities. Psychological Trauma: Theory, Research, Practice, and Policy. http://dx.doi.org/10.1037/tra0000781S133

Staub, E., \& Vollhardt, J. (2008). Altruism born of suffering: The roots of caring and helping after victimization and other trauma. American Journal of Orthopsychiatry, 78, 267-280

Tian, L., Du, M., \& Huebner, E. S. (2015). The effect of gratitude on elementary school students' subjective well-being in schools: The mediating role of prosocial behavior. Social Indicators Research, 122(3), 887-904. http://doi.org/10.1007/s11205-014-0712-9

Tian, L., Pi, L., Huebner, E. S., \& Du, M. (2016). Gratitude and adolescents' subjective well-being in school: The multiple mediating roles of basic psychological needs satisfaction at school. Frontiers in Psychology, 7, 1409. https://doi.org/10.3389/fpsyg.2016.01409

Trevelyan, E., Gambino, C., Gryn, T., Larsen, L., Acosta, Y., Grieco, E., et al. (2016, November). Characteristics of the U.S. population by generational status: 2013 (No. P23-214). Washington, DC: U.S. Census Bureau. Retrieved April 4, 2017, from https://www.census.gov/content/dam/Census/lib rary/publications/2016/demo/P23-214.pdf

van Agteren, J., Bartholomaeus, J., Fassnacht, D. B., Iasiello, M., Ali, K., Lo, L., \& Kyrios, M. (2020). Using Internet-Based Psychological Measurement to Capture the Deteriorating Community Mental Health Profile During COVID-19: Observational Study. JMIR Mental Health, 7(6), e20696. https://doi.org/10.2196/20696

Yang, D., Tu, C. C., \& Dai, X. (2020). The effect of the 2019 novel coronavirus pandemic on college students in Wuhan. Psychological Trauma: Theory, Research, Practice, and Policy, 12(S1), S6-S14. http://dx.doi.org/10.1037/tra0000930S6

Zheng, Y., Fan, Y., \& Lou, C. (2011). Relationship between gratitude and symptoms of post-traumatic stress disorder among adolescents: Mediation of social support and resilience. Psychological Development and Education, 5, 12.

Zhou, X., \& Wu, X. (2015). Longitudinal relationships between gratitude, deliberate rumination, and posttraumatic growth in adolescents following the Wenchuan earthquake in China. Scandinavian Journal of Psychology, 56, 567-572. http://doi.org/10.1111/sjop.12237 